\title{
MUTACIONES DEL CROMOSOMA X EN PACIENTES DEL SEXO FEMENINO DEL SERVICIO ACADÉMICO ASISTENCIAL DE GENÉTICA HUMANA DE LA UNMSM
}

\author{
Jaime DESCAILLEAUX y Margarita VELASQUEZ, \\ Laboratorio de Genética Humana, Facultad de Ciencias Biológicas, \\ UNMSM, CP. 170138 Lima - Perú; \\ Dirección: Telefax 464 9110, e -mail: jdescailleaux@unmsm.edu.pe
}

\section{RESUMEN}

Se ha estudiado citogenéticamente a 124 pacientes del sexo femenino que acudieron a la consulta médica por presentar una disfunción gonadal y/o un retardo del crecimiento. En 93 casos $(0,75)$ se encontró un cariotipo normal y en $31(0,25)$ un cariotipo anormal para el sexo femenino. Entre los cariotipos anormales la fórmula $45, \mathrm{X}$ con 16 casos fue la más frecuentemente encontrada $(0,52)$, seguida por el isocromosoma del brazo largo del $X$ que estuvo presente en 7 pacientes $(0,22)$, en 3 casos $(0,10)$, los cariotipos eran del tipo 46,XY y las pacientes afectadas por el síndrome de feminización testicular, en 2 casos $(0,06)$ un anillo del X, un caso $(0,03)$ era una rcpt equilibrada $(X ; X)(q 27 ; q 21)$ y los 2 restantes eran mosaicos con 2 líneas celulares. Entre los estigmas turnerianos registrados la implantación baja del cabello y el cubitus valgus fueron los más frecuentemente encontrados, mientras que la pequeña estatura y la disfunción ovárica siempre estuvieron presentes.

Palabras claves: Mutaciones cromosómicas, cromosoma X, disfunción gonadal., síndrome de Turner.

\section{ABSTRACT}

We shall report here the results of the cytogenetic analysis in 124 female patients that had visited the clinic Hospitals because in all the cases they had a gonadal disfunction and/or a deficit in height, 93 women had a normal karyotype (0.75) and $31(0.25)$ an abnormal karyotype. The monosomy of the X chromosome $45, X$ with 16 times $(0.52)$ were the more frequently among the abnormal karyotypes, followed by the isochromosome of the $\mathrm{X}$ long arm with 7 times (0.22), in 3 cases (0.10) the karyotypes were 46,XY and the patients affected by the testicular feminization syndrome, 2 cases $(0.06)$ it were a ring of the $X$, in 1 case $(0,03)$ a balanced rcpt $(X ; X)(q 27 ; q 21)$ and the two others were mosaics with 2 cellular lines. Among the turnerian stigmata the low set nucal hair and the cubitus valgus were the most frequently encountered, but the small stature and the gonadal disfunction were always present. 


\section{INTRODUCCIÓN}

La observación de que en algunas especies biológicas, principalmente en Drosophila, las variaciones numéricas de los cromosomas sexuales aparecían asociadas a diferentes estados disfuncionales de la diferenciación sexual y la fecundidad, levantó la hipótesis de que en la especie humana, también se registrarían casos patológicos similares, cuya etiología sería decurrente de una mutación cromosómica numérica o estructural, e impulsó a los genetistas a finales de la década del 50, a desarrollar la metodología del análisis citogenético con la finalidad de estudiar los cromosomas humanos y establecer sus variaciones normales y patológicas.

Una de las primeras variables cariotípicas descritas en el hombre, está representada por la monosomía de un cromosoma sexual $(45, \mathrm{X})$, tal como fuera reportada por Ford y col. en 1959, siendo este cariotipo, la anomalía cromosómica responsable por las características fenotípicas que se aprecian en las pacientes afectadas por el síndrome de Turner (Turner, 1938), y a la fecha, todo indica que se trata de la única monosomía viable de un cromosoma completo, en una línea celular homogénea.

En las pacientes afectadas por el síndrome de Turner, se puede observar una triada compuesta por: a). Estatura severamente disminuida $(132$ a $140 \mathrm{~cm})$ b). Infantilismo sexual como consecuencia de una disgenesia ovariana, encontrándose únicamente tejido fibroso en el lugar correspondiente a los ovarios, configurando una estructura que ha sido denominada como "gónadas en cinta" (streak gonads) y c). Una serie de malformaciones congénitas asociadas, que acompañan al síndrome en frecuencias relativas variables, figurando entre las más frecuentemente observadas las siguientes: cubitus valgus, cuello alado, cabello de implantación baja, dientes de mala implantación y maloclusión, orejas de implantación baja y malformadas, incremento de lunares (nevi) en la cara, pecho, espalda y extremidades, diámetro biacromial aumentado y diámetro pélvico disminuido, tórax ancho en forma de embudo, mamilas excesivamente separadas, linfedema en pies y manos que es muy notable al nacimiento, uñas hiperconvexas e hipoplásicas, ausencia de caracteres sexuales secundarios, etc., y a partir del trabajo de Ford y col., se considera la existencia de un cariotipo 45,X (o una variable), como un criterio determinante para el diagnóstico definitivo.

La frecuencia del síndrome de Turner es de aproximadamente 1 por cada 2500 niñas recién nacidas vivas (De Grouchy y Turleau, 1982), y la forma cariotípica 45,X es la que aparece con mayor frecuencia, esta cifra aparentemente no sufre modificaciones significativas en todas las poblaciones estudiadas, pero al lado de este cariotipo se ha reportado una serie de variables numéricas y estructurales, tanto en una línea celular homogénea, como en diferentes combinaciones que configuran mosaicismos cromosómicos con variaciones en la distribución de las líneas celulares, y que a su vez condicionan la variabilidad observada en la expresión de las diferentes malformaciones somáticas que acompañan al síndrome.

Sorprendentemente, el cariotipo $45, \mathrm{X}$ ha sido encontrado con una frecuencia relativa muy alta $(20 \%)$ entre los productos de los abortos espontáneos portadores de un cariotipo anormal (Hook, 1989, Dexeus y col., 1989); es importante recordar que las mutaciones que involucraban a los cromosomas sexuales son consideradas como las que menos severamente 
afectaban al hombre, en razón de la existencia de un mecanismo de compensación de dosis génica para los cromosomas X (Lyon 1961, 1988), siendo que en algunos mamíferos (ratones) la monosomía del cromosoma $\mathrm{X}$ no afectaba ni siquiera la fertilidad de las hembras portadoras del cariotipo "XO", las mismas que resultaban ser fértiles tan igual como las hembras XX, (Ashwort y col, 1991), así como también a la existencia de muy pocos genes mendelianos en el cromosoma $\mathrm{Y}$, excepción hecha de aquellos involucrados con la diferenciación testicular y la fecundidad masculina (Therman y Susman, 1996).

En esta ocasión, como una contribución al mejor conocimiento de la patología de los cromosomas sexuales que condicionan un fenotipo femenino en el Perú, presentamos los resultados obtenidos en un grupo de pacientes del sexo femenino, estudiadas en nuestro Laboratorio durante el período comprendido de 1991 a 1999, y que acudieron a la consulta médica a causa de una disfunción ovárica y/o retardo del crecimiento. Los objetivos que nos propusimos al iniciar este trabajo fueron los siguientes:

a.- Caracterizar los rearreglos estructurales del cromosoma $\mathrm{X}$ en la población de mujeres portadoras de disfunciones del ciclo menstrual y/o déficits del crecimiento que acuden al Servicio Académico Asistencial de Genética Humana. (SAAGH) de la UNMSM.

b.- Establecer una correlación cariotipo-fenotipo en la población femenina portadora de rearreglos del cromosoma $\mathrm{X}$, y de mosaicismos cariotípicos.

c.- Establecer la frecuencia de mutaciones numéricas y estructurales del cromosoma $\mathrm{X}$ en la población de pacientes del sexo femenino del SAAGH, que acudieron a la consulta médica por una disfunción gonadal y/o la presencia de estigmas turnerianos.

d.- Iniciar la formación de un centro referencial en el Perú sobre las características genéticas y citogenéticas de la población general.

\section{MATERIAL Y MÉTODOS}

El material biológico utilizado en esta ocasión, comprende un total de 124 pacientes del sexo femenino que llegaron al SAAGH, durante el período comprendido entre los años 1991 y 1999, con un diagnóstico primario de Síndrome de Turner, (exceptuando los tres casos de feminización testicular), y teniendo como motivo principal para la consulta médica, la existencia de un déficit del crecimiento y/o una disfunción ovariana, siendo que todos los casos eran procedentes de clínicas y centros hospitalarios de Lima Metropolitana.

En cada paciente se procedió a la toma de los datos y de la muestra considerando la siguiente secuencia: a). Colecta de información personal y familiar, según una ficha especialmente elaborada para este fin. b). Obtención de sangre periférica $(5-10 \mathrm{ml})$ en condiciones de asepsia, utilizando la heparina sódica como anticoagulante, con la finalidad de realizar el cultivo temporal de linfocitos y obtener cromosomas metafásicos o prometafásicos para el análisis cariotípico, y c). Raspado de la mucosa oral para la determinación de la cromatina sexual (cromatina $-\mathrm{X}$ ), incluyendo el análisis en prueba ciega. 
El cultivo de linfocitos fue realizado empleando la técnica de Moorhead y col., modificada para nuestro Laboratorio, las técnicas de coloración empleadas fueron la convencional con Giemsa, y para la obtención de cromosomas longitudinalmente diferenciados, en todos los casos utilizamos la denaturación térmica $\left(87^{\circ} \mathrm{C}\right)$ con la solución de Earle a pH 5,3 y 6,5 para la obtención de bandas $\mathrm{R}$ (RHG) según la técnica descrita por Dutrillaux y Couturier (1981), y para la marcación con bandas C (CBG) aplicamos el tratamiento con $\mathrm{Ba}(\mathrm{OH}) 2$ según la técnica de Sumner (1972) (ISCN, 1995).

El análisis citogenético se hizo en ampliaciones fotográficas, en cada paciente se analizó un número mínimo de 5 metafases marcadas por las técnicas RHG y CBG, o un número mayor según los casos, la aplicación de la coloración para bandas $\mathrm{C}$, nos permitió verificar la existencia de cromosomas dicéntricos con un centrómero inactivado, y adicionalmente, analizar la longitud de la heterocromatina constitutiva de los cromosomas 1, 9, y 16; La coloración y el análisis de la cromatina sexual se realizaron aplicando la técnica descrita por Descailleaux y Velásquez (1986), contándose un mínimo de 200 células de la mucosa oral, empleándose en todos los casos controles positivos y negativos.

\section{RESULTADOS}

Nuestros resultados se reportan en los cuadros numerados del 1 al 4, y en fotografías que ilustran algunos de los rearreglos reportados. En el cuadro $\mathrm{N}^{\circ} 1$, podemos apreciar que el total de pacientes estudiadas citogenéticamente asciende a 124, de ellas, 93 mostraron poseer un cariotipo normal para el sexo femenino $(46, \mathrm{XX})$ y representan el $75 \%$ de la muestra, mientras que en 31 casos encontramos un cariotipo anormal para el sexo femenino, lo que representa el $25 \%$ de la población, en todos los casos las pacientes acudieron a la consulta médica por presentar fundamentalmente un retardo del crecimiento y/o un cuadro de disfunción gonadal.

En el cuadro $\mathrm{N}^{\circ} 2$, se muestran todos los cariotipos anormales (31) encontrados en cada una de las pacientes estudiadas, así mismo, se reporta la información concerniente a la estatura, peso, edad, motivo de la consulta y la frecuencia relativa de células cromatino - positivas. (cromatina sexual - X).

En el cuadro $\mathrm{N}^{\circ} 3$, se presenta la distribución de los diferentes cariotipos anormales encontrados, indicando la existencia de líneas celulares únicas o en mosaicismos, se puede apreciar que el cariotipo $45, \mathrm{X}$ con 16 casos es el más frecuentemente encontrado correspondiendo a una frecuencia relativa algo mayor que el $52 \%$, y es seguido por el cariotipo $46, X Y$ con 3 casos (10\%), los mismos que han sido incluidos en la muestra porque a pesar de tratarse de un cariotipo normal, esta "normalidad" está referida al sexo masculino, y en el presente caso estamos tratando con pacientes que presentan un fenotipo femenino. Debemos indicar que en los 3 casos se trataba de pacientes afectados por el síndrome de feminización testicular (pseudohermafroditismo masculino), el mismo que es condicionado por una mutación de un gene responsable por la síntesis del receptor citosólico androgénico y que está ubicado en el cromosoma $X$. Los cariotipos restantes aparecen en números variables entre 1 y 2 casos, siendo que la aparición de isocromosomas del brazo largo del $\mathrm{X}$ ya sea al estado de 
isodicéntrico o monocéntrico, si fueran sumados apenas por el carácter de ser isocromosoma del brazo largo del $\mathrm{X}$ independientemente de existir en una línea celular única o en mosaicismos, llegarían a totalizar 7 casos que representan el $22 \%$ de la población con un cariotipo anormal. En el cuadro $\mathrm{N}^{\circ} 4$, se muestra la distribución de los estigmas turnerianos en relación a los diferentes cariotipos anormales encontrados, se puede observar que la estatura disminuida aparece en todos los cariotipos monosómicos para el cromosoma X total o parcial, en línea única o en mosaico, si bien se puede apreciar cierto grado de variabilidad en la disminución de la estatura, por ejemplo en todos los cariotipos 45,X la estatura está entre 5 y $6 \mathrm{~cm}$ por debajo de $140 \mathrm{~cm}$, excepto en dos casos que llegaron a $145 \mathrm{~cm}$ y en uno que alcanza a $140 \mathrm{~cm}$. También se puede percibir que una paciente que exhibe un isodicéntrico para el Xq alcanzó $145 \mathrm{~cm}$ y una con mosaicismo del tipo 45,X/46,XX alcanzó $143 \mathrm{~cm}$. (Ver también cuadro $\mathrm{N}^{\circ} 2$ ). La disfunción ovárica ha sido considerada solamente en las pacientes que tenían edad suficiente para apreciar la expresión de este carácter: (menarquia) en todas ellas aparece como un carácter igualmente constante.

Con relación a la distribución de las malformaciones congénitas asociadas al síndrome, podemos apreciar que la implantación baja del cabello, el cubitus valgus, la presencia de lunares en la cara, pecho y espalda, la ausencia de vello axilar y pubiano y el tórax en embudo, conforman, en el orden decreciente enunciado, los signos más frecuentemente observados, tanto en el cariotipo clásico del síndrome 45,X como en las variables cariotípicas reportadas.

El retardo del crecimiento fue la motivación fundamental para la consulta médica en las pacientes que tenían un rango de edad entre los 3 y 13 años, mientras que las que acudieron por ausencia de regla (amenorrea primaria) tenían una edad que fluctuaba entre los 14 y los 35 años.

En la figura $\mathrm{N}^{\mathbf{0}} 1$ presentamos algunas de las fotografías que nos permiten ilustrar los diferentes rearreglos estructurales encontrados del cromosoma $X$, se puede apreciar en a) un cromosoma $\mathrm{X}$ del tipo isodicèntrico [idic $(\mathrm{Xq})$ ], tratado con la técnica de marcación $\mathrm{CBG}$, mostrando que uno de los centrómeros permanece activo y el otro se volvió inactivo, mientras que en b) y c) se reporta una translocación recíproca ( $\mathrm{rcpt}$ ) entre los cromosomas $\mathrm{X}$ $[\operatorname{rcpt}(\mathrm{X} ; \mathrm{X})(\mathrm{q} 27 ; \mathrm{q} 21)]$, coloreada con las técnicas $\mathrm{RHG}$ y $\mathrm{CBG}$, y que corresponde también a un cromosoma dicéntrico, pero originado mediante una translocación recíproca entre los dos cromosomas $\mathrm{X}$, que registran puntos de quiebra en $\mathrm{q} 27$ y q21 respectivamente, seguido de una fusión que origina el cromosoma dicéntrico y luego la inactivación de uno de los centrómeros..

\section{DISCUSIÓN}

Los resultados obtenidos en las 124 pacientes estudiadas, nos muestran que sólo 31 eran portadoras de cariotipos anormales, es decir, que apenas en el $25 \%$ de los casos remitidos a nuestro Laboratorio, con sospecha de una mutación del cromosoma $\mathrm{X}$, se pudo confirmar el diagnóstico clínico, en los 93 casos restantes encontramos un cariotipo normal; este resultado nos indica que un porcentaje considerable de pacientes con problemas de crecimiento y/o amenorrea primaria $(75 \%)$, tienen como agente etiológico un factor diferente a la monosomía total o parcial del cromosoma $\mathrm{X}$. 
Entre los cariotipos anormales encontramos 21 con variaciones numéricas y 10 con rearreglos estructurales, observándose que el cariotipo $45, \mathrm{X}$ es el más frecuentemente encontrado con 16 casos sobre un total de 31 , aunque las cifras son aún pequeñas, este resultado es concordante con los resultados reportados previamente por diferentes autores (Hamerton, 1971, De Grouchy y Turleau, 1982, Hook 1989) que estimaron la frecuencia relativa del cariotipo 45,X en no menos de $155 \%$ del universo de las pacientes afectadas por el síndrome de Turner; las otras variables reportadas del cromosoma $X$, corresponden en 2 casos a mutaciones numéricas, y en 3 pacientes el cariotipo era 46,XY, con un diagnóstico clínico de feminización testicular y si bien podría ser discutible la inclusión de estas pacientes en este grupo, estimamos que ella se justifica si consideramos la causa por la cual se acude a la consulta médica que fue la ausencia de regla y/o déficit de crecimiento. (Ver cuadro 2).

Un análisis de las mutaciones estructurales reportadas, permiten apreciar la existencia de una super representación del rearreglo tipo isocromosoma del brazo largo del X, el cual aparece en 7 casos, ya sea bajo la forma de mono o dicéntrico, pero en ambos casos con un único centrómero funcional, en línea única o en mosaicos, este hallazgo constituye una evidencia para mostrar en primera instancia la existencia de centrómeros inactivos, y por ende un mecanismo inactivador de centrómeros que posibilita la segregación de las cromátides hermanas. (Ver fig.1a)

La ausencia de trabajos que reporten sobre la existencia de isocromosomas del brazo corto del $\mathrm{X}$, así como la demostración de isodicéntricos del brazo largo con un único centrómero inactivo, constituyen evidencias acerca de la génesis de estos rearreglos postulando la ocurrencia de una quiebra en la región del brazo corto inmediatamente proximal al centrómero, en una fase del ciclo celular previa a la síntesis de ADN, seguida por una soldadura en los mismos puntos de quiebra del brazo corto inmediatamente después de la duplicación del ADN y de esta forma se origina el isodicéntrico con una única cromátide, que se duplicará en el siguiente ciclo celular.

Los otros casos de rearreglos estructurales están representados por 2 pacientes que portan un cromosoma $\mathrm{X}$ en anillo, una en línea única y la otra bajo la forma de mosaico, y la restante tiene una translocación recíproca del tipo $\operatorname{rcpt}(\mathrm{X} ; \mathrm{X})(\mathrm{q} 27 ; \mathrm{q} 21)$, y que también ha conformado un cromosoma dicéntrico con un solo centrómero funcional apreciándose nítidamente la existencia del centrómero inactivo. (Ver fig. 1 b).

Si bien es verdad que teóricamente los rearreglos estructurales deberían condicionar un fenotipo menos severamente afectado que el cariotipo $45, \mathrm{X}$, nuestros resultados tienen aún cifras muy pequeñas y no nos permiten todavía emitir una opinión sobre este punto, consideramos que es prematuro intentar establecer una correlación cariotipo - fenotipo, y que es indispensable aumentar el número de casos de la muestra para poder alcanzar este objetivo.

Por otro lado, conociendo que en la gran mayoría de los casos no hay mayor dificultad para establecer con precisión el diagnóstico clínico del síndrome de Turner. Sin embargo, no debemos ignorar que la visita al médico se produce, en la gran mayoría de los casos, en la edad de la pubertad ante la ausencia de la menarquia, pocas veces durante la niñez por un déficit del crecimiento, mientras que en el recién nacido las posibilidades del diagnóstico precoz son muy escasas y están limitadas a signos de aparición menos frecuentes como son el exceso de piel en 
la nuca, linfedema en las extremidades y una disminución notable de la talla al nacimiento. (Massa y Vanderschueren - Lodeweyckx, 1991). Nuestros resultados son concordantes con resultados anteriormente reportados y que muestran la necesidad del análisis cariotípico como criterio determinante en el diagnóstico de la patología reproductiva femenina, la misma que está estrechamente relacionada con el desarrollo somático incluyendo la estatura normal.

Con respecto a la conformación de un centro referencial de pacientes con enfermedades genéticas y cromosómicas, con el número de pacientes estudiados estamos inaugurando nuestro Banco de Datos a partir de la información colectada, teniendo como uno de sus objetivos el establecimiento de correlaciones cariotipo-fenotipo. Igualmente estamos en condiciones de comentar que a la fecha nuestro Laboratorio está desarrollando un programa tendiente a caracterizar genéticamente a las poblaciones peruanas mediante la aplicación de marcadores moleculares y otros parámetros genéticos, que nos brindarán información sobre las características normales del hombre peruano y un mejor conocimiento de la Biología Humana (Jacobs y col, 1990).

\section{CONCLUSIONES:}

1. - En las 124 pacientes estudiadas en nuestro Servicio, $93(75 \%)$ son portadoras de un cariotipo normal y $31(25 \%)$ de un cariotipo anormal.

2. - Entre los 31 cariotipos anormales, la forma $45, \mathrm{X}$ es la más frecuentemente encontrada $(16 / 31=0,52)$.

3. - 23 de los 31 cariotipos anormales se presentaron en una única línea celular, y 8 bajo la forma de mosaicos.

4. - La pequeña estatura y disfunción ovariana son las características que aparecen en todos los casos de cariotipos anormales que involucran monosomías totales o parciales del cromosoma $\mathrm{X}$, siendo también verdadero que en algunos casos se observa variabilidad en la disminución de la estatura.

5. - Después del cariotipo $45, \mathrm{X}$, la formación de un isocromosoma, con uno o dos centrómeros en línea celular única o en mosaico, resulta ser con 7/31 casos la variable más frecuentemente encontrada entre los cariotipos anormales.,

6.- Los cariotipos $46, \mathrm{XY}$ que aparecieron en 3 pacientes afectados por el síndrome de la feminización testicular, son cariotipos normales para el sexo masculino, y no han sido considerados para el recuento de los estigmas turnerianos por cariotipo anormal.

AGRADECIMIENTOS: Los autores desean expresar su agradecimiento a todos los médicos que nos han brindado su confianza a lo largo de estos años en el análisis de sus pacientes y que mencionaremos en estricto orden alfabético; Alberto Allemant, Ana Alvarado, Oscar Castillo Sayán, Juan de Dios Cigueñas, Fausto Garmendia, Gloria Larrabure, César Olaya, Luis A. Orbegozo, Alfonso Ramirez, Rosario Rodríguez, María Rojas, José Sánchez, Cecilia Solís, Héctor Valdivia,

Asímismo, nuestro reconocimiento al FEDU - UNMSM (Fondo Especial de Desarrollo Universitario) por el apoyo económico brindado a nuestros proyectos. 
Cuadro $\mathbf{N}^{\circ}$ 1. Distribución de los Cariotipos examinados en 124 pacientes del sexo femenino del SAAGH de la UNMSM

\begin{tabular}{|l|l|l|}
\hline $\begin{array}{l}\text { CARIOTIPO } \\
\text { ENCONTRADOS }\end{array}$ & $\begin{array}{l}\text { FRECUENCIA } \\
\text { ABSOLUTA }\end{array}$ & $\begin{array}{l}\text { FRECUENCIA } \\
\text { RELATIVA }\end{array}$ \\
\hline NORMALES & 93 & 0,75 \\
\hline ANORMALES & 31 & 0,25 \\
\hline TOTAL & 124 & 1,00 \\
\hline
\end{tabular}

Cuadro $\mathbf{N}^{\circ}$ 2. Cariotipos Anormales encontrados en pacientes del sexo femenino del SAAGH de la UNMSM

\begin{tabular}{|c|c|c|c|c|c|c|}
\hline $\begin{array}{c}\mathrm{N}^{\circ} \\
\text { CASO }\end{array}$ & $\begin{array}{l}\text { EST } \\
(\mathrm{cm})\end{array}$ & $\begin{array}{l}\text { PES } \\
(\mathrm{kg})\end{array}$ & $\begin{array}{r}\text { EDAD } \\
(\mathbf{a n ̃ o s})\end{array}$ & $\begin{array}{l}\text { MOTIVO DE LA } \\
\text { CONSULTA }\end{array}$ & CARIOTIPO & $\begin{array}{c}\text { CROM-X } \\
(\%)\end{array}$ \\
\hline 1524 & 120 & 27 & 10 & Déficit crecimiento & $46, \mathrm{X}, \mathrm{i}(\mathrm{Xq}) / 45, \mathrm{X}$ & 14,5 \\
\hline 1535 & 119 & & 18 & AP. Fenotipo turneriano & $45, \mathrm{X}$ & 0 \\
\hline 1539 & 145 & & 18 & AP. No útero & $46, \mathrm{X}, \mathrm{idic}(\mathrm{Xq}) / 45, \mathrm{X}$ & 28. \\
\hline 1545 & 135 & & 15 & AP. Regla con tt h. & $45, \mathrm{X}$ & 0 \\
\hline 1583 & 145 & 46 & 19 & AP. Fenotipo turaeriano & $45, \mathrm{X}$ & 0 \\
\hline 1589 & & & 24 & $\mathrm{AP}$ & $46, X Y$ & 0 \\
\hline 1647 & 131 & 45 & 16 & AP. Fenotipo turneriano & $45, \mathrm{X}$ & 0 \\
\hline 1652 & 114 & & 10 & Def crecimi, dismorf discre & $46, \mathrm{X}, \mathrm{r}(\mathrm{X})$ & 0 \\
\hline 1666 & 130 & & 11 & Def crecimiento & $46, X, \mathrm{i}(\mathrm{Xq})$ & 29 \\
\hline 1672 & 132 & & 15 & AP. Fenotipo turneriano & $45, X$ & 0 \\
\hline 1677 & 131 & & 13 & No menarquia Fenotipo turne & $46, X, \operatorname{idic}(X q) / 45, X$ & 26 \\
\hline$\overline{1711}$ & 134 & 37 & 19 & AP. Fenotipo turneriano & $45, X$ & 0 \\
\hline 1715 & 135 & 37 & 25 & AP. .Fenotipo turneriano & $45, \mathrm{X}$ & 0 \\
\hline 1716 & 132 & 29 & 20 & AP. Fenotipo turneriano & $45, \mathrm{X}$ & 0 \\
\hline 1738 & 168 & 58 & 22 & AP. & $46, \mathrm{XX} / 47, \mathrm{XXX}$ & 24,5 \\
\hline 1760 & 134 & 42 & 18 & AP. Fenotipo turneriano & $45, X / 46, X, i(X q)$ & 0 \\
\hline 1782 & 140 & - & 17 & AP. Fenotipo turneriano & $45, \mathrm{X}$ & 0 \\
\hline 1798 & 143 & 39,5 & 23 & AP. Dismorfia.discreta & $45 \mathrm{X} / 46, \mathrm{XX}$ & 0 \\
\hline 1837 & $13 \mathrm{l}$ & 40 & 12 & Def crecimie Dism discreta & $46, X, \mathrm{i}(\mathrm{Xq})$ & 25 \\
\hline 1845 & 120 & - & 13 & No menarquia Fenotipo turne & $45, \mathrm{X}$ & 0 \\
\hline 1852 & 132 & - & 19 & AP. Fenotipo turneriano & $45, \mathrm{X}$ & 0 \\
\hline 1876 & 135 & 41 & 30 & AP. Fenotipo turneriano & $45, \mathrm{X}$ & 0 \\
\hline 1888 & 130 & 25 & 11 & Def. crecim. No fenot turneri & $46, \mathrm{XX} / 46, \mathrm{X}, \mathrm{i}(\mathrm{Xg})$ & \\
\hline 1897 & 110 & 21 & 8 & Def. crecimiento & $45, \mathrm{X}$ & 0 \\
\hline 1910 & 145 & 41,5 & 19 & AP. Dism discreta & $45, \mathrm{X}$ & 0 \\
\hline 1915 & 139 & - & 17 & AP. Fenotipo turneriano & $45, \mathrm{X} / 46, \mathrm{X}, \mathrm{r}(\mathrm{X})$ & - \\
\hline 1974 & 156 & 49 & 18 & AP. & $46, X Y$ & 0 \\
\hline 1976 & 141 & 51 & 18 & AP. Fenotipo tumeriano & $46, X, \operatorname{dic}(X), t(X q 27 ; X q 21)$ & 0 \\
\hline 1996 & 119 & 27 & 12 & No menarquia Fenotipo tume & $45, \mathrm{X}$ & 0 \\
\hline 2018 & 152 & 45 & 20 & AP. & $46, X Y$ & 0 \\
\hline 2024 & 51 & 3.29 & 1 mes & Linfedema, piel extra cuello & $45, \mathrm{X}, 16 \mathrm{qh}+$ & 0 \\
\hline
\end{tabular}

$\mathrm{EST}=$ Estatura

$\mathrm{PES}=$ Peso

$\mathrm{T}$ th $=$ Tratamiento hormonal
Dism = dismorfia

$\mathrm{AP} \quad=$ Amenorrea primaria 
Cuadro $\mathbf{N}^{\circ}$ 3. Distribución de los Cariotipos Anormales de las pacientes del sexo femenino del SAAGH de la UNMSM

\begin{tabular}{|l|l|l|l|}
\hline \multicolumn{1}{|c|}{$\begin{array}{c}\text { CARIOTIPOS } \\
\text { ENCONTRADOS }\end{array}$} & \multicolumn{1}{|c|}{$\begin{array}{c}\mathbf{N}^{\circ} \text { DE } \\
\text { CASOS }\end{array}$} & $\begin{array}{c}\text { FRECUENCIA } \\
\text { RELATIVA }\end{array}$ & $\begin{array}{c}\text { DISTRIBUCIÓN DE } \\
\text { LÍNEA CELULAR }\end{array}$ \\
\hline $45, \mathrm{X}$ & 16 & 0,52 & HOMOGENEA \\
\hline $46, \mathrm{XY}$ & 3 & 0,10 & HOMOGENEA \\
\hline $46, \mathrm{X}, \mathrm{i}(\mathrm{Xq})$ & 2 & 0,06 & HOMOGENEA \\
\hline $46, \mathrm{X}, \mathrm{r}(\mathrm{X})$ & 1 & 0,03 & HOMOGENEA \\
\hline $46, \mathrm{X}, \mathrm{dic}(\mathrm{X}) \mathrm{t}(\mathrm{Xq} 27 ; \mathrm{Xq} 21)$ & 1 & 0,03 & HOMOGENEA \\
\hline $45, \mathrm{X} / 46, \mathrm{X}, \mathrm{i}(\mathrm{Xq})$ & 2 & 0,06 & MOSAICO \\
\hline $45, \mathrm{X} / 46, \mathrm{X}, \mathrm{idic}(\mathrm{Xq})$ & 2 & 0,06 & MOSAICO \\
\hline $45, \mathrm{X} / 46, \mathrm{XX}$ & 1 & 0,03 & MOSAICO \\
\hline $46, \mathrm{XX} / 46, \mathrm{X}, \mathrm{i}(\mathrm{Xq})$ & 1 & 0,03 & MOSAICO \\
\hline $45, \mathrm{X} / 46, \mathrm{X}, \mathrm{r}(\mathrm{X})$ & 1 & 0,03 & MOSAICO \\
\hline $46, \mathrm{XX} / 47, \mathrm{XXX}$ & 1 & 0,03 & MOSAICO \\
\hline $\mathrm{TOTAL}$ & 31 & 0,98 & \\
\hline
\end{tabular}

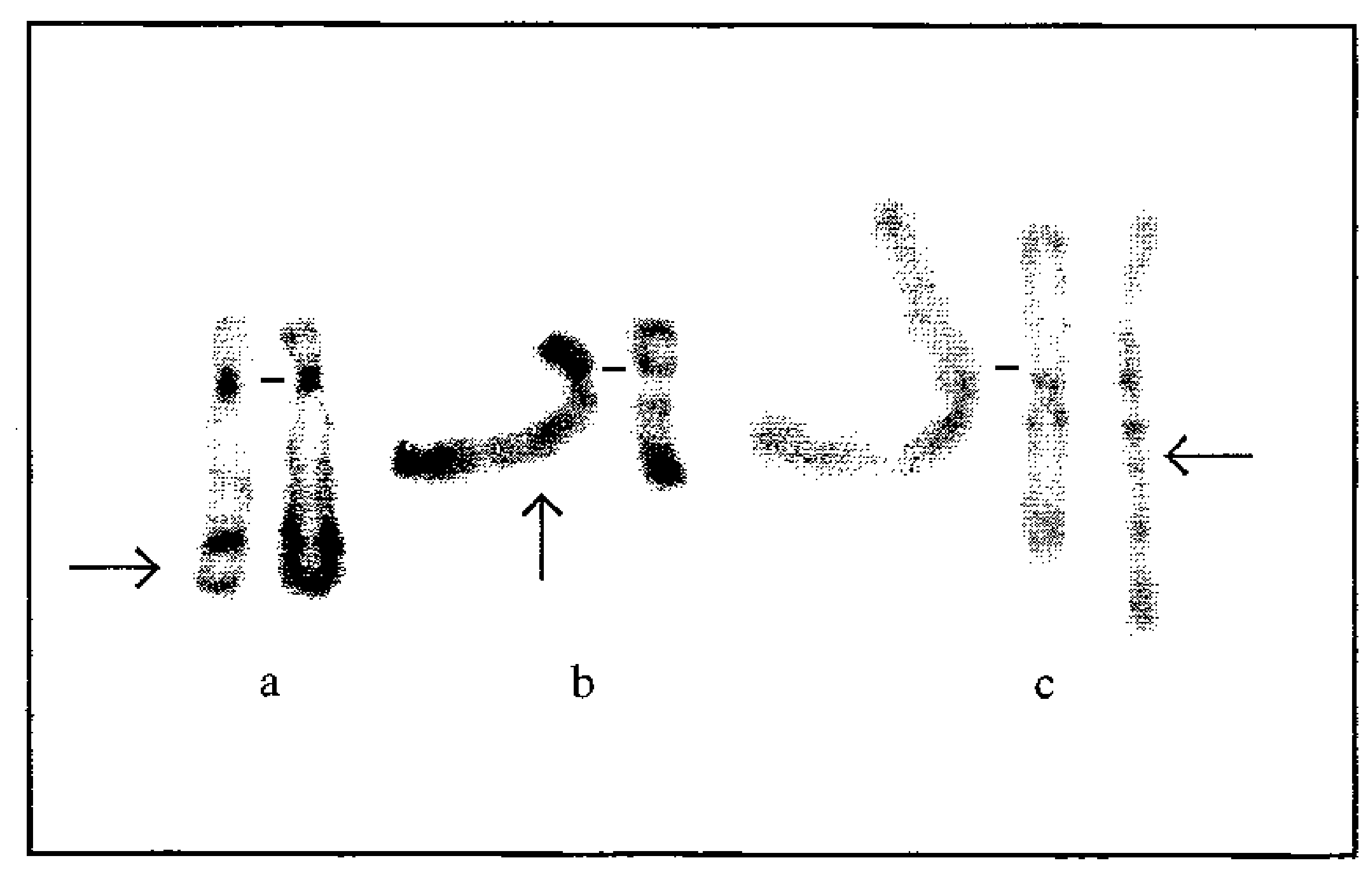

Fig $N^{\circ}$ 1.- a). der del $X$ con un centrómero inactivo, $C B G$.

b). der del $X$ mostrando los puntos de quiebra y soldadura (q27;q21) RHG.

c). idic del Xq mostrando el centrómero inactivo $C B G$. 

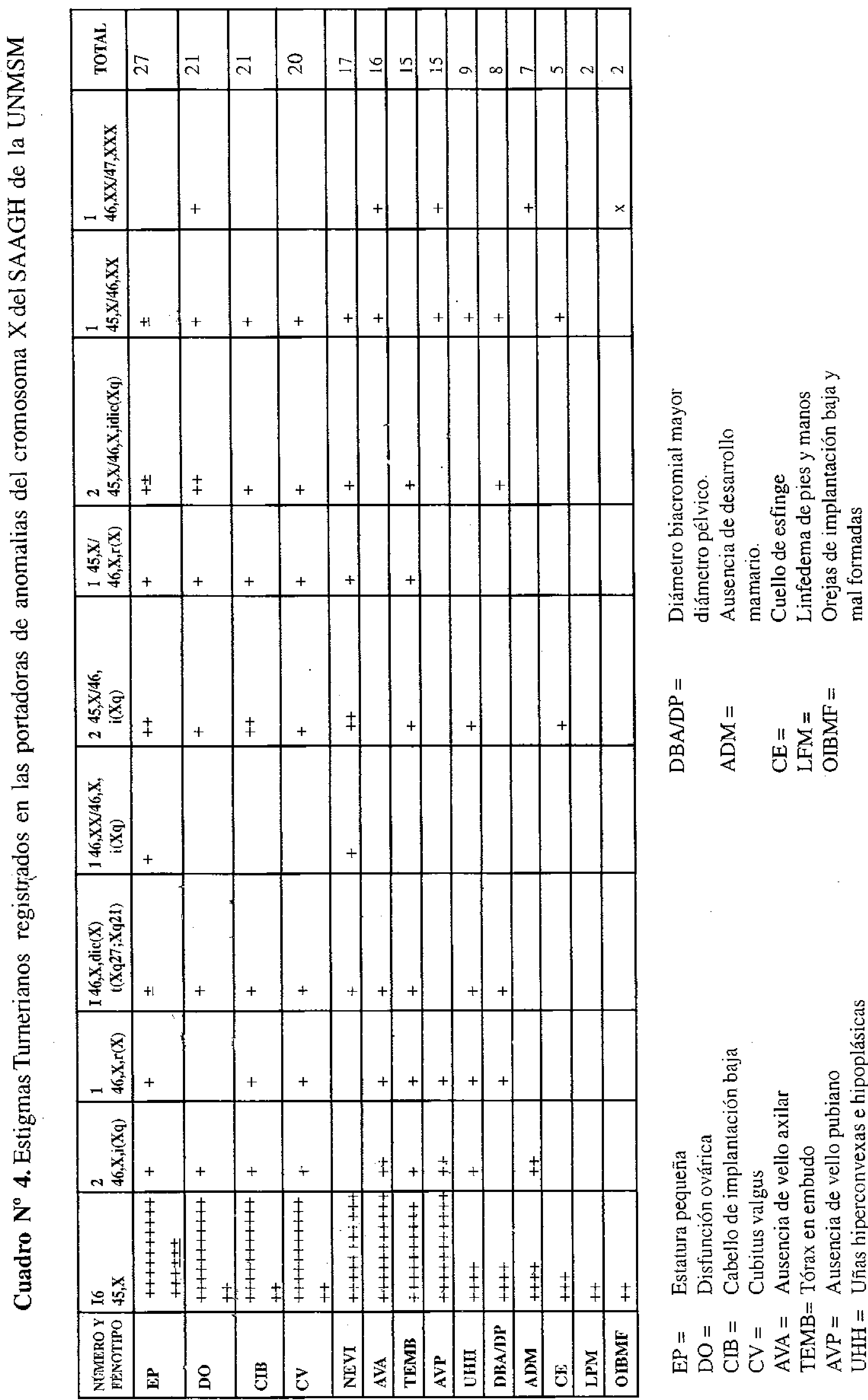

E

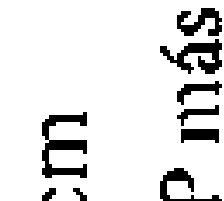
용 पृ $\stackrel{0}{0}$ $\stackrel{5}{\Xi}$ 守 的

愛 票总

๘

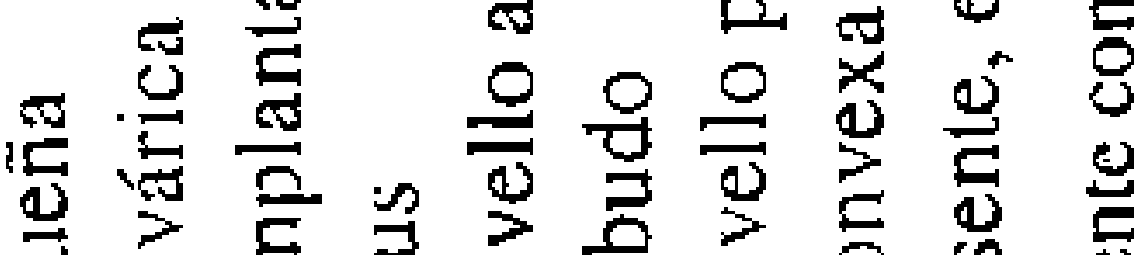
可 हᄐ

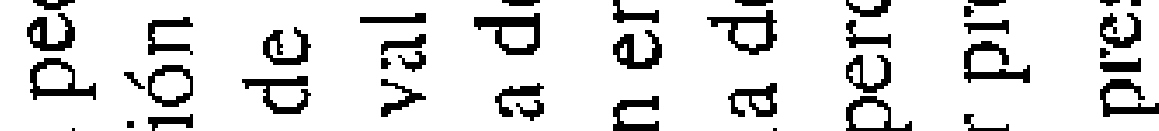

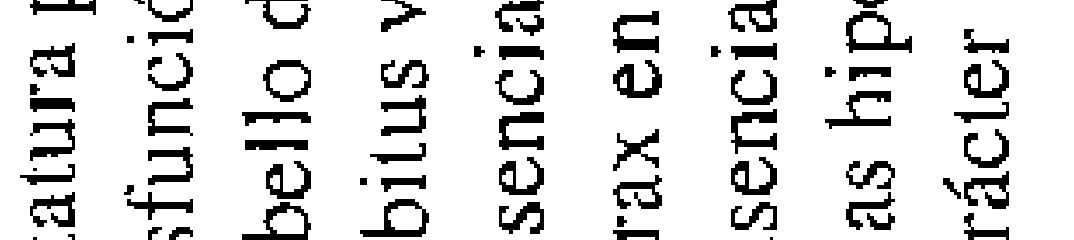

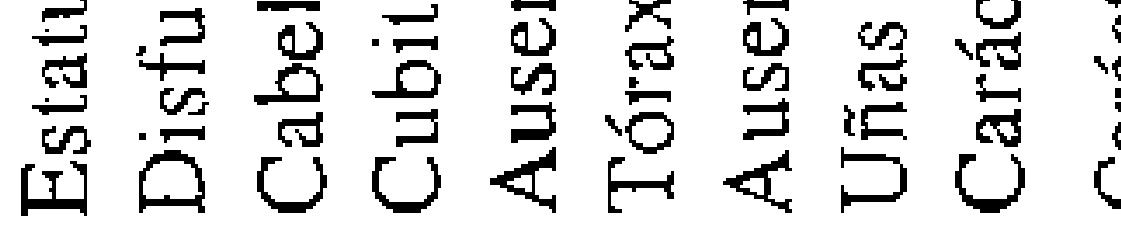

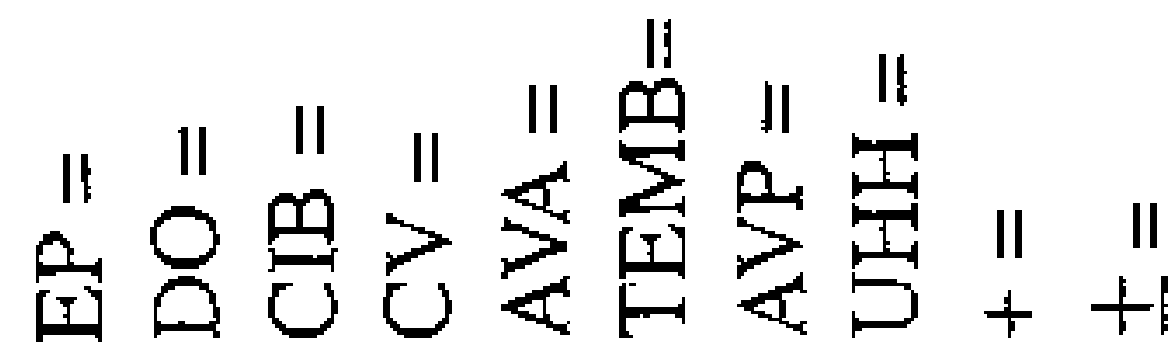




\section{REFERENCIAS BIBLIOGRÁFICAS:}

1.- ASHWORT A., RASTAN S., LOVELL-BADGE R., KAY G. (1991). X chromosome inactivation may explain the difference in viability of XO humans and mice. Nature, 351:406-408.

2.- $\quad$ BENDER BG, HARMON RJ, LINDEN MG, ROBINSON A. (I995). Psychosocial adaptation of 39 adolescents with sex chromosome abnormalities. Pediatrics Abstracts, 96, 2, 302-308.

3.- DESCALLEAUX J y VELASQUEZM. (1986). «Una técnica simplificada para cromatina sexual» Rev. Ciencias, 74, 1; pp. 171-177. UNMSM (Lima).

4.- DEXEUS S, CARRERA JM, ALEGRE M, SALVADOR C., SOLE MT, (1989). El riesgo de nacer. El desafío del diagnóstico prenatal. Ed Labor SA.. Barcelona.

5.- DUtTRLLAUX B. et COUTURIER J. (1981). La pratique de l'analyse chromosomique. Ed. Masson, París.

6.- $\quad$ EORD CE., JONES KW., POLANI PE.,ALMEIDA JC., BRIGGS JH. (1959). A sex chromosome anomalie in a case of gonadal dysgenesis (Turner's syndrome). Lancet, i: 711-713.

7.- GROUCHY J. de, TURLEAUC., (1982). Atlas des maladies chromosomiques., 2me. edition. Expansion scientifique française, pp 376-393. París.

8.- HAMERTON JL., (1971).: Human Cytogenetics: Clinical Cytogenetics, Vol. 1I, New York, Academic Press, pp.. 65-112.

9.- HOOK HB., (1989). Epidemiologic and design aspects of studies of somatic chromosome breakage and sister-chromatid exchange. In: Mutation epidemiologic: Review and Recommendations. Committee 5 Report. ICPEMC. Elsevier Science Publishers. Amsterdam.

10.- ISCN: (1995): AN INTERNATIONAL SYSTEM EOR HUMAN CYTOGENETIC NOMENCLATURE. Mitelman F (ed); S KARGER, BASEL, 1995.

11.- JACOBS PA, BETTS PR, COCKWELL AE y Col. (1990). A cytogenetical and molecular reappraisal of a series of patients with Turner's syndrome. Ann Hum Genet. 54: 209-223

12.- LYON MF. (1961). Gene action in the X chromosome of the mouse. Nature. 190: $372-373$.

13.- LYON MF. (1988). X-chromosome inactivation and the location and expression of X_linked genes. Am J Hum Genet. 42: 8 - 16.

14.- MAVEL A., TURC C., FELDMAN JP., MICHIELS Y., NIVELON-CHEVALIER A., KOLOWSKI JP., GERVAIS G. (1980). La fonction gonadique des femmes à caryotypes XO homogène ou en mosaique numérique. J. Gynecol. Obstet. Biol. Reprod., 9:875-886.

15.- MASSA GG., VANDERSCHUEREN - LODEWEYCKX M. (1991) Age and height at diagnosis in Turnersyndrome: influence of parental height. Pediatrics Abstracts, 88,6, 1148-1152.

16.- THERMAN E, SUSMAN M. (1996). Cromosomas humanos, estructura, comportamiento y efectos. Publicado por: Sociedade Brasileira de Genética, traducción, Drets M. Brasil.

17.- TURNER HH., (1938). A syndrome of infantilism, congenital webbed neck and cubitus valgus. Endocrinology, 23: 266-574. 